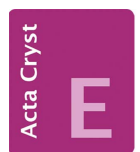

CRYSTALLOGRAPHIC COMMUNICATIONS

ISSN 2056-9890

Received 31 August 2016

Accepted 28 September 2016

Edited by W. T. A. Harrison, University of Aberdeen, Scotland

Keywords: crystal structure; $\pi-\pi$ interactions; $\mathrm{C}-\mathrm{H} \cdots \pi$ interactions; chromane; quantumchemical calculations.

CCDC reference: 1507161

Supporting information: this article has supporting information at journals.iucr.org/e

\section{Crystal structure of 2-oxo-2H-chromen-3-yl propanoate}

\author{
Eric Ziki, ${ }^{a *}$ Jules Yoda, ${ }^{b}$ Abdoulaye Djandé, ${ }^{b}$ Adama Saba ${ }^{b}$ and Rita Kakou-Yao ${ }^{a}$ \\ aLaboratoire de Cristallographie et Physique Moléculaire, UFR SSMT, Université Félix Houphouët Boigny de Cocody 22 \\ BP 582 Abidjan 22, Côte d'Ivoire, and 'baboratoire de Chimie Moléculaire et Matériaux, Equipe de Chimie Organique et \\ Phytochimie, Université Ouaga I Pr Joseph KI-ZERBO 03 BP 7021 Ouagadougou 03, Burkina Faso. *Correspondence \\ e-mail: eric.ziki@gmail.com
}

In the title compound, $\mathrm{C}_{12} \mathrm{H}_{10} \mathrm{O}_{4}$, the dihedral angle between the coumarin ring system [maximum deviation $=0.033(8) \AA$ ] and the propionate side chain is $78.48(8)^{\circ}$. In the crystal, weak $\mathrm{C}-\mathrm{H} \cdots \mathrm{O}$ hydrogen bonds generate inversion dimers and and $\mathrm{C}-\mathrm{H} \cdots \pi$ and $\pi-\pi$ interactions link the dimers into a threedimensional network. A quantum chemical calculation is in good agreement with the observed structure.

\section{Chemical context}

Coumarin and its derivatives are widely recognized for their multiple biological activities, including anticancer (Lacy et al., 2004; Kostova, 2005), anti-inflammatory (Todeschini et al., 1998), antiviral (Borges et al., 2005), antimalarial (Agarwal et al., 2005) and anticoagulant (Maurer et al., 1998) properties. As part of our studies in this area, we now describe the synthesis and crystal structure of the title compound, (I).

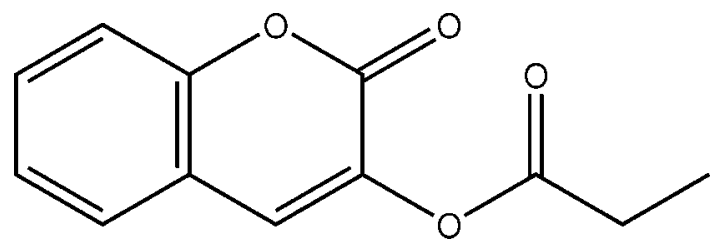

\section{Structural commentary}

In compound (I) (Fig. 1), the coumarin ring system is almost planar [maximum deviation $=0.033(1) \AA]$ and is oriented at an angle of $70.84(8)^{\circ}$ with respect to the plane formed by the

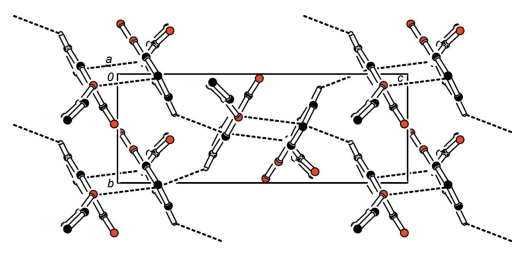

OPEN $\odot$ ACCESS
Figure 1

The molecular structure of compound (I), with displacement ellipsoids drawn at the $50 \%$ probability level.

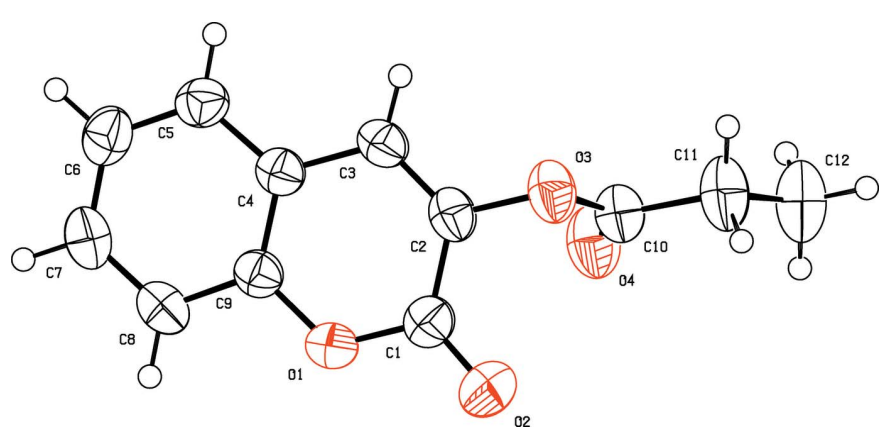




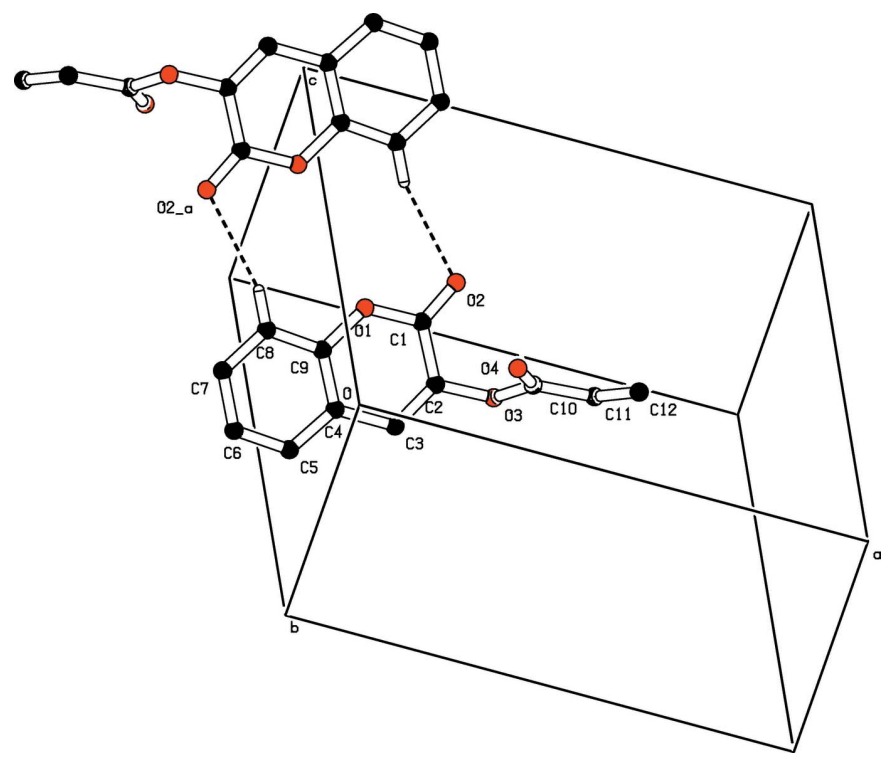

Figure 2

View of an inversion dimer linked by a pair of $\mathrm{C} 8-\mathrm{H} 8 \cdots \mathrm{O} 2(-x,-y$, $-z+1)$ interactions, generating an $R_{2}^{2}(12)$ loop. This dimers stack by unit translation along the $c$ axis. $\mathrm{H}$ atoms not involved in hydrogen bonding have been omitted.

propanoate group. An inspection of the bond lengths shows that there is a slight asymmetry of the electronic distribution around the coumarin ring: the $\mathrm{C} 2-\mathrm{C} 3[1.329$ (2) $\AA]$ and $\mathrm{C} 2-$ C1 [1.460 (2) $\AA$ ] bond lengths are shorter and longer, respectively, than those expected for a $\mathrm{C}_{\mathrm{ar}}-\mathrm{C}_{\mathrm{ar}}$ bond. This suggests that the electron density is preferentially located in the $\mathrm{C} 2-\mathrm{C} 3$ bond at the pyrone ring, as seen in other coumarin-3-carboxamide derivatives (Gomes et al., 2016).

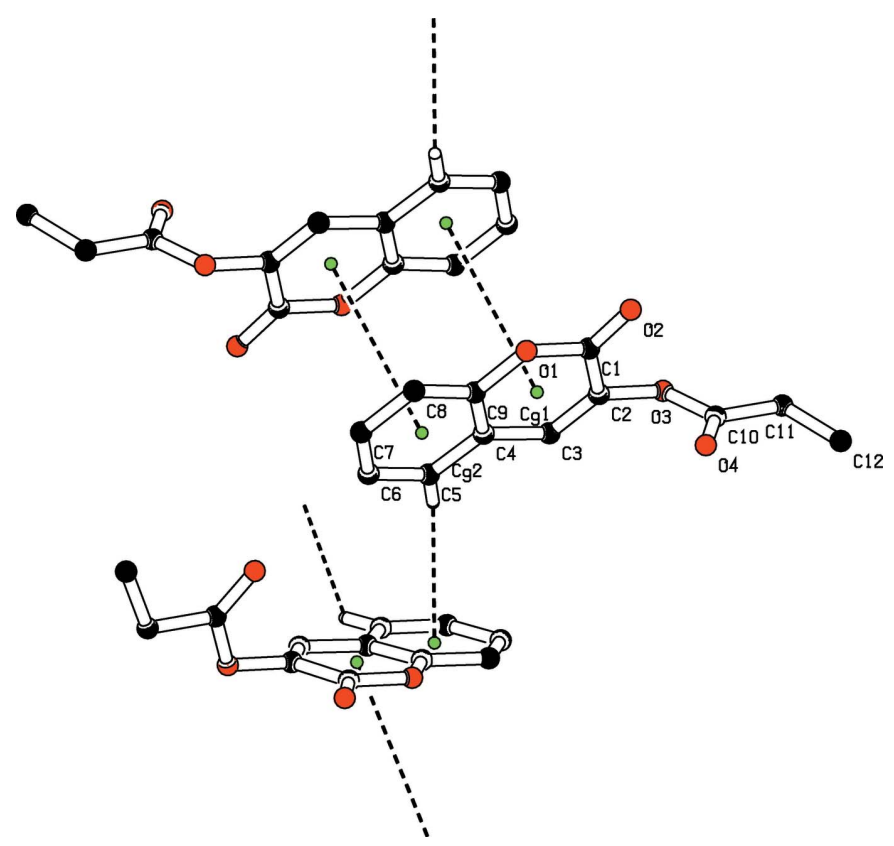

Figure 3

A view of the crystal packing, showing the $\pi-\pi$ stacking and $\mathrm{C}-\mathrm{H} \cdots \pi$ interactions (dashed lines). The green dots are ring centroids. $\mathrm{H}$ atoms not involved in the $\mathrm{C}-\mathrm{H} \cdots \pi$ interactions have been omitted for clarity.
Table 1

Hydrogen-bond geometry $\left(\AA,^{\circ}\right)$.

$\mathrm{Cg} 2$ is the centroid of the $\mathrm{C} 4-\mathrm{C} 9$ ring.

\begin{tabular}{lllll}
\hline$D-\mathrm{H} \cdots A$ & $D-\mathrm{H}$ & $\mathrm{H} \cdots A$ & $D \cdots A$ & $D-\mathrm{H} \cdots A$ \\
\hline $\mathrm{C} 8-\mathrm{H} 8 \cdots \mathrm{O} 2^{\mathrm{i}}$ & 0.93 & 2.59 & $3.4783(19)$ & 161 \\
$\mathrm{C} 5-\mathrm{H} 5 \cdots C g 2^{\mathrm{ii}}$ & 0.93 & 2.78 & $3.4959(16)$ & 134 \\
\hline
\end{tabular}

Symmetry codes: (i) $-x,-y,-z+1$; (ii) $-x, y+\frac{1}{2},-z+\frac{1}{2}$.

\section{Supramolecular features}

In the crystal, the molecules are linked by pairs of $\mathrm{C} 8-$ $\mathrm{H} 8 \cdots \mathrm{O} 2(x,-y, 1-z)$ weak hydrogen bonds to form $R_{2}^{2}(12)$ loops, which lie in a chain running along the $c$ axis direction (Fig. 2). Weak aromatic $\pi-\pi$ stacking interactions of 3.7956 (8) $\AA$ (Janiak, 2000) are present between the coumarin pyran ring (centroid $C g 1$ ) and benzene ring (centroid $C g 2$ ) of symmetry-related $(-x, 1-y, 1-z)$ molecules, thus forming a three-dimensional supramolecular network. A weak C$\mathrm{H} \cdots C g$ ( $\pi$-ring) interaction is also present (Figs. 3 and 4, and Table 1).

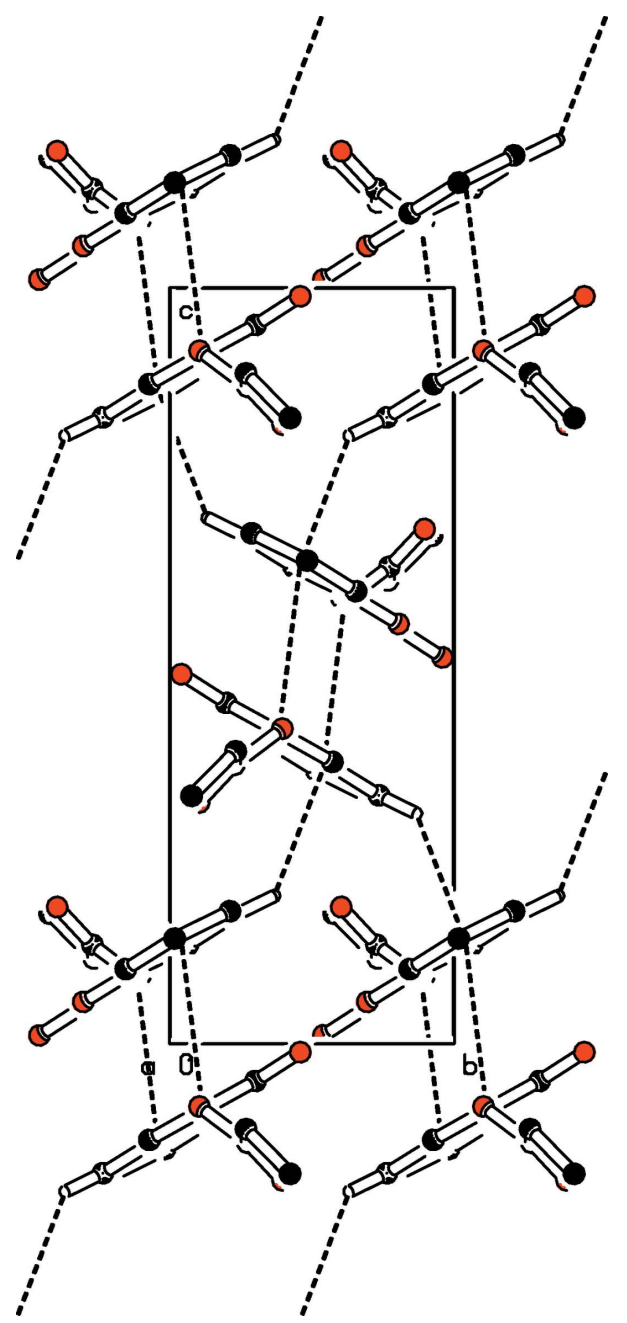

Figure 4

Part of the crystal structure of (I), showing $\mathrm{C}-\mathrm{H} \cdots \pi$ and $\pi-\pi$ interactions as dashed lines. $\mathrm{H}$ atoms have been omitted for clarity. 
Table 2

Experimental and calculated bond lengths $(\AA)$.

\begin{tabular}{lll}
\hline Bond & X-ray & HF(6-31G) \\
\hline O1-C1 & $1.3628(17)$ & 1.371 \\
O1-C9 & $1.3769(17)$ & 1.378 \\
O2-C1 & $1.2004(18)$ & 1.227 \\
O3-C10 & $1.3713(18)$ & 1.359 \\
O3-C2 & $1.3893(17)$ & 1.381 \\
O4-C10 & $1.1932(19)$ & 1.21 \\
C1-C2 & $1.460(2)$ & 1.468 \\
C2-C3 & $1.329(2)$ & 1.355 \\
C3-C4 & $1.4403(19)$ & 1.441 \\
C4-C5 & $1.401(2)$ & 1.406 \\
C4-C9 & $1.3928(18)$ & 1.407 \\
C5-C6 & $1.370(2)$ & 1.387 \\
C6-C7 6705 \\
C7-C8 & $1.386(2)$ & 1.383 \\
C8-C9 & $1.379(2)$ & 1.408 \\
C10-C11 & $1.3842(19)$ & 1.497 \\
C11-C12 & $1.495(2)$ & 1.525 \\
\hline
\end{tabular}

\section{Theoretical calculations}

Quantum-chemical calculations were performed to compare with the experimental analysis. An ab-initio Hartree-Fock (HF) method was used with the standard basis set of 6-31G using the GAUSSIAN03 software package (Frisch et al., 2004; Dennington et al., 2007) to obtain the optimized molecular structure. The computational results are in good agreement with the experimental crystallographic data (Table 2).

\section{Synthesis and crystallization}

In a $100 \mathrm{ml}$ round-necked flask topped with a water condenser were introduced successively $25 \mathrm{ml}$ of dried diethyl ether, 6.17 $\times 10^{-3} \mathrm{~mol}(\simeq 0.8 \mathrm{ml})$ of propionic anhydride and $2.35 \mathrm{ml}(4.7$ molar equivalents) of dried pyridine. While stirring strongly, $6.17 \times 10^{-3} \mathrm{~mol}(1 \mathrm{~g})$ of 3 -hydroxycoumarin was added in small portions over $30 \mathrm{~min}$. The reaction mixture was left under agitation at room temperature for $3 \mathrm{~h}$. The mixture was then poured in a separating funnel containing $40 \mathrm{ml}$ of chloroform and washed with diluted hydrochloric acid solution until the $\mathrm{pH}$ was $2-3$. The organic layer was extracted, washed with water to neutrality, dried over $\mathrm{MgSO}_{4}$ and the solvent removed. The resulting precipitate (crude product) was filtered off with petroleum ether and recrystallized from a solvent mixture of chloroform-hexane $(1 / 3, v / v)$. Colourless prisms of the title compound were obtained in a yield of $65 \%$, m. p. $=351-353 \mathrm{~K}$.

\section{Refinement}

Crystal data, data collection and structure refinement details are summarized in Table 3. $\mathrm{H}$ atoms were placed in calculated positions $[\mathrm{C}-\mathrm{H}=0.93$ (aromatic), 0.96 (methyl) or $0.97 \AA$ (methylene)] and refined using a riding-model approximation with $U_{\text {iso }}(\mathrm{H})$ constrained to 1.2 (aromatic and methylene group) or 1.5 (methyl group) times $U_{\text {eq }}$ of the respective parent atom.
Table 3

Experimental details.

\begin{tabular}{|c|c|}
\hline \multicolumn{2}{|l|}{ Crystal data } \\
\hline Chemical formula & $\mathrm{C}_{12} \mathrm{H}_{10} \mathrm{O}_{4}$ \\
\hline$M_{\mathrm{r}}$ & 218.20 \\
\hline Crystal system, space group & Monoclinic, $P 2_{1} / c$ \\
\hline Temperature $(\mathrm{K})$ & 293 \\
\hline$a, b, c(\AA)$ & $12.1179(4), 5.7243(2), 15.3275(5)$ \\
\hline$\beta\left(^{\circ}\right)$ & $94.881(3)$ \\
\hline$V\left(\AA^{3}\right)$ & $1059.36(6)$ \\
\hline$Z$ & 4 \\
\hline Radiation type & $\mathrm{Cu} K \alpha$ \\
\hline$\mu\left(\mathrm{mm}^{-1}\right)$ & 0.87 \\
\hline Crystal size $(\mathrm{mm})$ & $0.46 \times 0.16 \times 0.08$ \\
\hline \multicolumn{2}{|l|}{ Data collection } \\
\hline Diffractometer & $\begin{array}{l}\text { Agilent SuperNova Dual }(\mathrm{Cu} \text { at } \\
\text { zero) Source diffractometer with } \\
\text { an AtlasS2 detector }\end{array}$ \\
\hline Absorption correction & $\begin{array}{l}\text { Multi-scan (CrysAlis PRO; } \\
\text { Agilent, 2014) }\end{array}$ \\
\hline$T_{\min }, T_{\max }$ & $0.778,1.000$ \\
\hline $\begin{array}{l}\text { No. of measured, independent and } \\
\text { observed }[I>2 \sigma(I)] \text { reflections }\end{array}$ & $6028,1930,1655$ \\
\hline$R_{\text {int }}$ & 0.020 \\
\hline$(\sin \theta / \lambda)_{\max }\left(\AA^{-1}\right)$ & 0.605 \\
\hline \multicolumn{2}{|l|}{ Refinement } \\
\hline$R\left[F^{2}>2 \sigma\left(F^{2}\right)\right], w R\left(F^{2}\right), S$ & $0.038,0.117,1.06$ \\
\hline No. of reflections & 1930 \\
\hline No. of parameters & 145 \\
\hline H-atom treatment & $\mathrm{H}$-atom parameters constrained \\
\hline$\Delta \rho_{\max }, \Delta \rho_{\min }\left(\mathrm{e} \AA^{-3}\right)$ & $0.16,-0.16$ \\
\hline
\end{tabular}

Computer programs: CrysAlis PRO (Agilent, 2014), SHELXS97 (Sheldrick, 2008), SHELXL2013 (Sheldrick, 2015), PLATON (Spek, 2009) and publCIF (Westrip, 2010).

\section{Acknowledgements}

The authors thank the Spectropole Service of the faculty of Sciences (Aix-Marseille, France) for the use of the diffractometer and the NMR and MS spectrometers.

\section{References}

Agarwal, A., Srivastava, K., Puri, S. K. \& Chauhan, P. M. S. (2005). Bioorg. Med. Chem. 13, 4645-4650.

Agilent. (2014). CrysAlis PRO. Agilent Technologies Ltd, Yarnton, England.

Borges, F., Roleira, F., Milhazes, N., Santana, L. \& Uriarte, E. (2005). Curr. Med. Chem. 12, 887-916.

Dennington, R., Keith, T. \& Millam, J. (2007). Gaussview4.1. Semichem Inc., Shawnee Mission, KS, USA.

Frisch, M. J., et al. (2004). GAUSSIAN03. Gaussian Inc., Wallingford, CT, USA.

Gomes, L. R., Low, J. N., Fonseca, A., Matos, M. J. \& Borges, F. (2016). Acta Cryst. E72, 926-932.

Janiak, C. (2000). J. Chem. Soc. Dalton Trans. pp. 3885-3896.

Kostova, I. (2005). Curr. Med. Chem. Anticancer Agents, 5, 29-46.

Lacy, A. \& O'Kennedy, R. (2004). Curr. Pharm. Des. 10, 3797-3811.

Maurer, H. H. \& Arlt, J. W. (1998). J. Chromatogr. B Biomed. Sci. Appl. 714, 181-195.

Sheldrick, G. M. (2008). Acta Cryst. A64, 112-122.

Sheldrick, G. M. (2015). Acta Cryst. C71, 3-8.

Spek, A. L. (2009). Acta Cryst. D65, 148-155.

Todeschini, A. R., de Miranda, A. L. P., da Silva, K. C. M., Parrini, S. C. \& Barreiro, E. J. (1998). Eur. J. Med. Chem. 33, 189-199.

Westrip, S. P. (2010). J. Appl. Cryst. 43, 920-925. 


\section{supporting information}

Acta Cryst. (2016). E72, 1562-1564 [https://doi.org/10.1107/S2056989016015279]

\section{Crystal structure of 2-oxo-2H-chromen-3-yl propanoate}

\section{Eric Ziki, Jules Yoda, Abdoulaye Djandé, Adama Saba and Rita Kakou-Yao}

Computing details

Data collection: CrysAlis PRO (Agilent, 2014); cell refinement: CrysAlis PRO (Agilent, 2014); data reduction: CrysAlis PRO (Agilent, 2014); program(s) used to solve structure: SHELXS97 (Sheldrick, 2008); program(s) used to refine structure: SHELXL2013 (Sheldrick, 2015); molecular graphics: PLATON (Spek, 2009); software used to prepare material for publication: publCIF (Westrip, 2010).

2-Oxo-2H-chromen-3-yl propanoate

Crystal data

$\mathrm{C}_{12} \mathrm{H}_{10} \mathrm{O}_{4}$

$M_{r}=218.20$

Monoclinic, $P 2_{1} / c$

Hall symbol: $-\mathrm{P} 2 \mathrm{ybc}$

$a=12.1179$ (4) $\AA$

$b=5.7243(2) \AA$

$c=15.3275(5) \AA$

$\beta=94.881(3)^{\circ}$

$V=1059.36(6) \AA^{3}$

$Z=4$

\section{Data collection}

Agilent SuperNova Dual (Cu at zero) Source diffractometer with an AtlasS2 detector

Radiation source: sealed X-ray tube

Mirror monochromator

Detector resolution: 5.3048 pixels $\mathrm{mm}^{-1}$

$\omega$ scan

Absorption correction: multi-scan

(CrysAlis PRO; Agilent, 2014)

$T_{\min }=0.778, T_{\max }=1.000$

Refinement

Refinement on $F^{2}$

Least-squares matrix: full

$R\left[F^{2}>2 \sigma\left(F^{2}\right)\right]=0.038$

$w R\left(F^{2}\right)=0.117$

$S=1.06$

1930 reflections

145 parameters

0 restraints

40 constraints
$F(000)=456$

$D_{\mathrm{x}}=1.368 \mathrm{Mg} \mathrm{m}^{-3}$

Melting point: $351 \mathrm{~K}$

$\mathrm{Cu} K \alpha$ radiation, $\lambda=1.54184 \AA$

Cell parameters from 3028 reflections

$\theta=5.8-68.6^{\circ}$

$\mu=0.87 \mathrm{~mm}^{-1}$

$T=293 \mathrm{~K}$

Prism, colourless

$0.46 \times 0.16 \times 0.08 \mathrm{~mm}$

6028 measured reflections

1930 independent reflections

1655 reflections with $I>2 \sigma(I)$

$R_{\text {int }}=0.020$

$\theta_{\text {max }}=68.9^{\circ}, \theta_{\text {min }}=3.7^{\circ}$

$h=-14 \rightarrow 14$

$k=-6 \rightarrow 6$

$l=-15 \rightarrow 18$

Primary atom site location: structure-invariant direct methods

Secondary atom site location: difference Fourier map

Hydrogen site location: inferred from neighbouring sites

$\mathrm{H}$-atom parameters constrained

$w=1 /\left[\sigma^{2}\left(F_{\mathrm{o}}^{2}\right)+(0.0631 P)^{2}+0.1085 P\right]$

where $P=\left(F_{\mathrm{o}}^{2}+2 F_{\mathrm{c}}{ }^{2}\right) / 3$ 
$(\Delta / \sigma)_{\max }<0.001$

$\Delta \rho_{\max }=0.16$ e $\AA^{-3}$

$\Delta \rho_{\min }=-0.16$ e $\AA^{-3}$

Special details

Geometry. All esds (except the esd in the dihedral angle between two 1.s. planes) are estimated using the full covariance matrix. The cell esds are taken into account individually in the estimation of esds in distances, angles and torsion angles; correlations between esds in cell parameters are only used when they are defined by crystal symmetry. An approximate (isotropic) treatment of cell esds is used for estimating esds involving l.s. planes.

Fractional atomic coordinates and isotropic or equivalent isotropic displacement parameters $\left(\AA^{2}\right)$

\begin{tabular}{lllll}
\hline & $x$ & $y$ & $z$ & $U_{\text {iso }} * / U_{\text {eq }}$ \\
\hline O1 & $0.08845(8)$ & $0.19504(17)$ & $0.44462(6)$ & $0.0505(3)$ \\
O3 & $0.36752(8)$ & $0.39585(19)$ & $0.41680(7)$ & $0.0567(3)$ \\
C9 & $0.02550(11)$ & $0.3708(2)$ & $0.40435(8)$ & $0.0426(3)$ \\
C2 & $0.25245(11)$ & $0.3964(2)$ & $0.40998(9)$ & $0.0466(3)$ \\
C3 & $0.19407(11)$ & $0.5719(2)$ & $0.37284(8)$ & $0.0455(3)$ \\
H3 & 0.2298 & 0.6985 & 0.3497 & $0.055^{*}$ \\
C4 & $0.07494(11)$ & $0.5644(2)$ & $0.36879(8)$ & $0.0420(3)$ \\
C8 & $-0.08834(12)$ & $0.3450(3)$ & $0.40123(9)$ & $0.0521(3)$ \\
H8 & -0.1198 & 0.2150 & 0.4258 & $0.063 *$ \\
C1 & $0.20130(12)$ & $0.1982(2)$ & $0.45088(9)$ & $0.0492(3)$ \\
O4 & $0.36176(9)$ & $0.1032(2)$ & $0.31915(8)$ & $0.0683(3)$ \\
O2 & $0.24985(10)$ & $0.0406(2)$ & $0.48893(8)$ & $0.0695(3)$ \\
C5 & $0.00553(12)$ & $0.7361(2)$ & $0.32863(9)$ & $0.0497(3)$ \\
H5 & 0.0362 & 0.8676 & 0.3045 & $0.060^{*}$ \\
C6 & $-0.10727(13)$ & $0.7114(3)$ & $0.32463(10)$ & $0.0566(4)$ \\
H6 & -0.1527 & 0.8257 & 0.2975 & $0.068^{*}$ \\
C7 & $-0.15398(12)$ & $0.5169(3)$ & $0.36080(10)$ & $0.0567(4)$ \\
H7 & -0.2306 & 0.5022 & 0.3578 & $0.068^{*}$ \\
C10 & $0.41542(12)$ & $0.2272(3)$ & $0.36892(10)$ & $0.0530(3)$ \\
C11 & $0.53858(13)$ & $0.2315(4)$ & $0.38698(13)$ & $0.0728(5)$ \\
H11A & 0.5644 & 0.3905 & 0.3808 & $0.087^{*}$ \\
H11B & 0.5576 & 0.1839 & 0.4471 & $0.087^{*}$ \\
C12 & $0.59736(16)$ & $0.0768(5)$ & $0.32791(15)$ & $0.0858(6)$ \\
H12A & 0.6758 & 0.0871 & 0.3428 & $0.129^{*}$ \\
H12B & 0.5806 & 0.1252 & 0.2683 & $0.129^{*}$ \\
H12C & 0.5736 & -0.0817 & 0.3346 & $0.129 *$ \\
& & & &
\end{tabular}

Atomic displacement parameters $\left(\AA^{2}\right)$

\begin{tabular}{lllllll}
\hline & $U^{11}$ & $U^{22}$ & $U^{33}$ & $U^{12}$ & $U^{13}$ & $U^{23}$ \\
\hline O1 & $0.0500(5)$ & $0.0463(5)$ & $0.0557(5)$ & $-0.0037(4)$ & $0.0068(4)$ & $0.0091(4)$ \\
O3 & $0.0398(5)$ & $0.0614(6)$ & $0.0686(6)$ & $-0.0025(4)$ & $0.0019(4)$ & $-0.0145(5)$ \\
C9 & $0.0453(7)$ & $0.0438(6)$ & $0.0394(6)$ & $-0.0015(5)$ & $0.0069(5)$ & $-0.0014(5)$ \\
C2 & $0.0395(7)$ & $0.0505(7)$ & $0.0499(7)$ & $-0.0036(5)$ & $0.0052(5)$ & $-0.0083(5)$ \\
C3 & $0.0468(7)$ & $0.0425(7)$ & $0.0485(7)$ & $-0.0072(5)$ & $0.0109(5)$ & $-0.0037(5)$ \\
C4 & $0.0459(7)$ & $0.0411(6)$ & $0.0398(6)$ & $-0.0018(5)$ & $0.0083(5)$ & $-0.0037(5)$ \\
C8 & $0.0473(7)$ & $0.0570(8)$ & $0.0535(7)$ & $-0.0083(6)$ & $0.0125(6)$ & $-0.0022(6)$
\end{tabular}


supporting information

\begin{tabular}{lllllll} 
C1 & $0.0506(7)$ & $0.0490(7)$ & $0.0479(7)$ & $0.0031(6)$ & $0.0034(5)$ & $0.0009(6)$ \\
O4 & $0.0510(6)$ & $0.0805(8)$ & $0.0730(7)$ & $-0.0006(5)$ & $0.0025(5)$ & $-0.0238(6)$ \\
O2 & $0.0665(7)$ & $0.0669(7)$ & $0.0749(7)$ & $0.0122(6)$ & $0.0041(6)$ & $0.0197(6)$ \\
C5 & $0.0575(8)$ & $0.0450(7)$ & $0.0474(7)$ & $0.0025(6)$ & $0.0100(6)$ & $0.0013(5)$ \\
C6 & $0.0548(8)$ & $0.0611(9)$ & $0.0540(7)$ & $0.0145(7)$ & $0.0065(6)$ & $-0.0010(6)$ \\
C7 & $0.0411(7)$ & $0.0715(9)$ & $0.0584(8)$ & $0.0027(6)$ & $0.0097(6)$ & $-0.0069(7)$ \\
C10 & $0.0450(8)$ & $0.0594(8)$ & $0.0545(7)$ & $0.0007(6)$ & $0.0038(6)$ & $-0.0053(6)$ \\
C11 & $0.0430(8)$ & $0.0917(13)$ & $0.0829(11)$ & $0.0053(8)$ & $-0.0003(7)$ & $-0.0168(10)$ \\
C12 & $0.0526(10)$ & $0.1094(16)$ & $0.0951(13)$ & $0.0179(10)$ & $0.0050(9)$ & $-0.0168(12)$ \\
\hline
\end{tabular}

Geometric parameters $\left(\AA,{ }^{\circ}\right)$

\begin{tabular}{|c|c|c|c|}
\hline $\mathrm{O} 1-\mathrm{C} 1$ & $1.3628(17)$ & $\mathrm{O} 4-\mathrm{C} 10$ & $1.1932(19)$ \\
\hline $\mathrm{O} 1-\mathrm{C} 9$ & $1.3769(17)$ & $\mathrm{C} 5-\mathrm{C} 6$ & $1.370(2)$ \\
\hline $\mathrm{O} 3-\mathrm{C} 10$ & $1.3713(18)$ & $\mathrm{C} 5-\mathrm{H} 5$ & 0.9300 \\
\hline $\mathrm{O} 3-\mathrm{C} 2$ & $1.3893(17)$ & $\mathrm{C} 6-\mathrm{C} 7$ & $1.386(2)$ \\
\hline $\mathrm{C} 9-\mathrm{C} 8$ & $1.3842(19)$ & $\mathrm{C} 6-\mathrm{H} 6$ & 0.9300 \\
\hline $\mathrm{C} 9-\mathrm{C} 4$ & $1.3926(18)$ & $\mathrm{C} 7-\mathrm{H} 7$ & 0.9300 \\
\hline $\mathrm{C} 2-\mathrm{C} 3$ & $1.329(2)$ & $\mathrm{C} 10-\mathrm{C} 11$ & $1.495(2)$ \\
\hline $\mathrm{C} 2-\mathrm{C} 1$ & $1.460(2)$ & $\mathrm{C} 11-\mathrm{C} 12$ & $1.491(3)$ \\
\hline $\mathrm{C} 3-\mathrm{C} 4$ & $1.4403(19)$ & $\mathrm{C} 11-\mathrm{H} 11 \mathrm{~A}$ & 0.9700 \\
\hline $\mathrm{C} 3-\mathrm{H} 3$ & 0.9300 & C11-H11B & 0.9700 \\
\hline $\mathrm{C} 4-\mathrm{C} 5$ & $1.401(2)$ & $\mathrm{C} 12-\mathrm{H} 12 \mathrm{~A}$ & 0.9600 \\
\hline $\mathrm{C} 8-\mathrm{C} 7$ & $1.379(2)$ & $\mathrm{C} 12-\mathrm{H} 12 \mathrm{~B}$ & 0.9600 \\
\hline $\mathrm{C} 8-\mathrm{H} 8$ & 0.9300 & $\mathrm{C} 12-\mathrm{H} 12 \mathrm{C}$ & 0.9600 \\
\hline $\mathrm{C} 1-\mathrm{O} 2$ & $1.2004(18)$ & & \\
\hline $\mathrm{C} 1-\mathrm{O} 1-\mathrm{C} 9$ & $122.43(10)$ & $\mathrm{C} 4-\mathrm{C} 5-\mathrm{H} 5$ & 119.8 \\
\hline $\mathrm{C} 10-\mathrm{O} 3-\mathrm{C} 2$ & $115.41(11)$ & $\mathrm{C} 5-\mathrm{C} 6-\mathrm{C} 7$ & $120.31(14)$ \\
\hline $\mathrm{O} 1-\mathrm{C} 9-\mathrm{C} 8$ & $116.74(12)$ & $\mathrm{C} 5-\mathrm{C} 6-\mathrm{H} 6$ & 119.8 \\
\hline $\mathrm{O} 1-\mathrm{C} 9-\mathrm{C} 4$ & $121.11(12)$ & $\mathrm{C} 7-\mathrm{C} 6-\mathrm{H} 6$ & 119.8 \\
\hline $\mathrm{C} 8-\mathrm{C} 9-\mathrm{C} 4$ & $122.15(13)$ & $\mathrm{C} 8-\mathrm{C} 7-\mathrm{C} 6$ & $120.90(14)$ \\
\hline $\mathrm{C} 3-\mathrm{C} 2-\mathrm{O} 3$ & $121.88(12)$ & $\mathrm{C} 8-\mathrm{C} 7-\mathrm{H} 7$ & 119.6 \\
\hline $\mathrm{C} 3-\mathrm{C} 2-\mathrm{C} 1$ & $122.80(12)$ & $\mathrm{C} 6-\mathrm{C} 7-\mathrm{H} 7$ & 119.6 \\
\hline $\mathrm{O} 3-\mathrm{C} 2-\mathrm{C} 1$ & $115.22(12)$ & $\mathrm{O} 4-\mathrm{C} 10-\mathrm{O} 3$ & $121.89(13)$ \\
\hline $\mathrm{C} 2-\mathrm{C} 3-\mathrm{C} 4$ & $119.40(12)$ & $\mathrm{O} 4-\mathrm{C} 10-\mathrm{C} 11$ & $127.56(15)$ \\
\hline $\mathrm{C} 2-\mathrm{C} 3-\mathrm{H} 3$ & 120.3 & $\mathrm{O} 3-\mathrm{C} 10-\mathrm{C} 11$ & $110.52(13)$ \\
\hline $\mathrm{C} 4-\mathrm{C} 3-\mathrm{H} 3$ & 120.3 & $\mathrm{C} 12-\mathrm{C} 11-\mathrm{C} 10$ & $113.50(15)$ \\
\hline $\mathrm{C} 9-\mathrm{C} 4-\mathrm{C} 5$ & $117.88(12)$ & $\mathrm{C} 12-\mathrm{C} 11-\mathrm{H} 11 \mathrm{~A}$ & 108.9 \\
\hline $\mathrm{C} 9-\mathrm{C} 4-\mathrm{C} 3$ & $118.03(12)$ & $\mathrm{C} 10-\mathrm{C} 11-\mathrm{H} 11 \mathrm{~A}$ & 108.9 \\
\hline $\mathrm{C} 5-\mathrm{C} 4-\mathrm{C} 3$ & $124.05(12)$ & $\mathrm{C} 12-\mathrm{C} 11-\mathrm{H} 11 \mathrm{~B}$ & 108.9 \\
\hline $\mathrm{C} 7-\mathrm{C} 8-\mathrm{C} 9$ & $118.32(13)$ & $\mathrm{C} 10-\mathrm{C} 11-\mathrm{H} 11 \mathrm{~B}$ & 108.9 \\
\hline $\mathrm{C} 7-\mathrm{C} 8-\mathrm{H} 8$ & 120.8 & $\mathrm{H} 11 \mathrm{~A}-\mathrm{C} 11-\mathrm{H} 11 \mathrm{~B}$ & 107.7 \\
\hline $\mathrm{C} 9-\mathrm{C} 8-\mathrm{H} 8$ & 120.8 & $\mathrm{C} 11-\mathrm{C} 12-\mathrm{H} 12 \mathrm{~A}$ & 109.5 \\
\hline $\mathrm{O} 2-\mathrm{C} 1-\mathrm{O} 1$ & $118.14(13)$ & $\mathrm{C} 11-\mathrm{C} 12-\mathrm{H} 12 \mathrm{~B}$ & 109.5 \\
\hline $\mathrm{O} 2-\mathrm{C} 1-\mathrm{C} 2$ & $125.74(14)$ & $\mathrm{H} 12 \mathrm{~A}-\mathrm{C} 12-\mathrm{H} 12 \mathrm{~B}$ & 109.5 \\
\hline $\mathrm{O} 1-\mathrm{C} 1-\mathrm{C} 2$ & $116.12(12)$ & $\mathrm{C} 11-\mathrm{C} 12-\mathrm{H} 12 \mathrm{C}$ & 109.5 \\
\hline $\mathrm{C} 6-\mathrm{C} 5-\mathrm{C} 4$ & $120.43(13)$ & $\mathrm{H} 12 \mathrm{~A}-\mathrm{C} 12-\mathrm{H} 12 \mathrm{C}$ & 109.5 \\
\hline
\end{tabular}




$\begin{array}{llll}\mathrm{C} 6-\mathrm{C} 5-\mathrm{H} 5 & 119.8 & \mathrm{H} 12 \mathrm{~B}-\mathrm{C} 12-\mathrm{H} 12 \mathrm{C} & 109.5 \\ \mathrm{C} 1-\mathrm{O} 1-\mathrm{C} 9-\mathrm{C} 8 & 179.07(12) & \mathrm{C} 9-\mathrm{O} 1-\mathrm{C} 1-\mathrm{C} 2 & -1.73(18) \\ \mathrm{C} 1-\mathrm{O} 1-\mathrm{C} 9-\mathrm{C} 4 & \mathrm{C} 3-\mathrm{C} 2-\mathrm{C} 1-\mathrm{O} 2 & 0.3(2) \\ \mathrm{C} 10-\mathrm{O} 3-\mathrm{C} 2-\mathrm{C} 3 & \mathrm{O} 3-\mathrm{C} 2-\mathrm{C} 1-\mathrm{O} 2 & 3.69(19) \\ \mathrm{C} 10-\mathrm{O} 3-\mathrm{C} 2-\mathrm{C} 1 & \mathrm{C} 3-\mathrm{C} 2-\mathrm{C} 1-\mathrm{O} 1 & -179.78(10) \\ \mathrm{O} 3-\mathrm{C} 2-\mathrm{C} 3-\mathrm{C} 4 & \mathrm{O} 3-\mathrm{C} 2-\mathrm{C} 1-\mathrm{O} 1 & -0.27(19) \\ \mathrm{C} 1-\mathrm{C} 2-\mathrm{C} 3-\mathrm{C} 4 & -179.09(11) & \mathrm{C} 9-\mathrm{C} 4-\mathrm{C} 5-\mathrm{C} 6 & 177.46(12) \\ \mathrm{O} 1-\mathrm{C} 9-\mathrm{C} 4-\mathrm{C} 5 & -2.8(2) & \mathrm{C} 3-\mathrm{C} 4-\mathrm{C} 5-\mathrm{C} 6 & 0.4(2) \\ \mathrm{C} 8-\mathrm{C} 9-\mathrm{C} 4-\mathrm{C} 5 & 179.85(11) & \mathrm{C} 9-\mathrm{C} 8-\mathrm{C} 7-\mathrm{C} 6 & -0.4(2) \\ \mathrm{O} 1-\mathrm{C} 9-\mathrm{C} 4-\mathrm{C} 3 & -0.21(19) & \mathrm{C} 5-\mathrm{C} 6-\mathrm{C} 7-\mathrm{C} 8 & 6.2(2) \\ \mathrm{C} 8-\mathrm{C} 9-\mathrm{C} 4-\mathrm{C} 3 & 1.98(17) & \mathrm{C} 2-\mathrm{O} 3-\mathrm{C} 10-\mathrm{O} 4 & -175.47(14) \\ \mathrm{C} 2-\mathrm{C} 3-\mathrm{C} 4-\mathrm{C} 9 & -178.08(12) & \mathrm{C} 2-\mathrm{O} 3-\mathrm{C} 10-\mathrm{C} 11 & 6.5(3) \\ \mathrm{C} 2-\mathrm{C} 3-\mathrm{C} 4-\mathrm{C} 5 & -0.08(18) & \mathrm{O} 4-\mathrm{C} 10-\mathrm{C} 11-\mathrm{C} 12 & -171.65(17) \\ \mathrm{O} 1-\mathrm{C} 9-\mathrm{C} 8-\mathrm{C} 7 & -177.81(12) & \mathrm{O} 3-\mathrm{C} 10-\mathrm{C} 11-\mathrm{C} 12 & \\ \mathrm{C} 4-\mathrm{C} 9-\mathrm{C} 8-\mathrm{C} 7 & -179.53(12) & & \\ \mathrm{C} 9-\mathrm{O} 1-\mathrm{C} 1-\mathrm{O} 2 & 0.5(2) & 178.23(13) & \end{array}$

Hydrogen-bond geometry $\left(A,{ }^{\circ}\right)$

$\mathrm{Cg} 2$ is the centroid of the $\mathrm{C} 4-\mathrm{C} 9$ ring.

\begin{tabular}{lllll}
\hline$D-\mathrm{H} \cdots A$ & $D-\mathrm{H}$ & $\mathrm{H} \cdots A$ & $D \cdots A$ & $D-\mathrm{H} \cdots A$ \\
\hline $\mathrm{C} 8-\mathrm{H} 8 \cdots \mathrm{O} 2^{\mathrm{i}}$ & 0.93 & 2.59 & $3.4783(19)$ & 161 \\
$\mathrm{C} 5-\mathrm{H} 5 \cdots C g 2^{\mathrm{ii}}$ & 0.93 & 2.78 & $3.4959(16)$ & 134 \\
\hline
\end{tabular}

Symmetry codes: (i) $-x,-y,-z+1$; (ii) $-x, y+1 / 2,-z+1 / 2$. 